\title{
NORMAL FORMS FOR HYPERSURFACES OF FINITE TYPE IN $\mathbb{C}^{2}$
}

\author{
Martin KolÁř
}

\begin{abstract}
We construct normal forms for Levi degenerate hypersurfaces of finite type in $\mathbb{C}^{2}$. As one consequence, an explicit solution to the problem of local biholomorphic equivalence is obtained. Another consequence determines the dimension of the stability group of the hypersurface.
\end{abstract}

\section{Introduction}

Levi degenerate hypersurfaces have been intensively studied since the pioneering work of J. J. Kohn $([\mathrm{K}])$, which introduced the concept of finite type. On the one hand, there is now deep qualitative theory which links local geometry and analysis on pseudoconvex domains of finite type (see [CS] for references). On the other hand, some fundamental quantitative results from the nondegenerate case still did not find analogy (cf. [BFG]).

Our aim is to show that the construction of normal forms, developed by J. Moser in $[\mathrm{CM}]$, has a natural generalization to Levi degenerate hypersurfaces of finite type in dimension two. As a consequence, the problem of local biholomorphic equivalence of two hypersurfaces is reduced to a low dimensional algebraic calculation. In fact, the remaining algebra behind normal forms is much simpler than in the nondegenerate case, as there are less symmetries of the model hypersurface. Another application gives precise information on the dimension of the stability group of the hyperfurface at the given point.

Being the main motivation, we first formulate the local equivalence problem. Let $M_{1}, M_{2} \subseteq \mathbb{C}^{n}$ be real analytic hypersurfaces of real codimension one and $p_{1}, p_{2}$ be points on $M_{1}$ and $M_{2}$, respectively. If there is an invertible holomorphic map $f$ defined in a neighbourhood $U$ of $p_{1}$ such that $f\left(p_{1}\right)=p_{2}$ and $f\left(M_{1} \cap U\right) \subseteq$ $M_{2}$, the two hypersurfaces are said to be locally biholomorphically equivalent. The problem is to find a complete, computable set of local invariants, which provide a way to recognize equivalent hypersurfaces.

The history of the problem starts with H. Poincaré, who showed that the Riemann mapping theorem has no equivalent in higher dimensions, and nontrivial local invariants do exist. For $n=2$, a solution was found by E. Cartan in $[\mathrm{C} 1],[\mathrm{C} 2]$, under the assumption that the hypersurfaces are regular, i.e., the

Received by the editors October 15, 2004.

Supported by a grant of the GA ČR no. 201/05/2117. 
Levi form is nondegenerate at $p_{1}, p_{2}$. For $n \geq 2$, with the same regularity assumption, the problem was solved by Chern and Moser. While the result of the second part of $[\mathrm{CM}]$ generalizes Cartan's result to higher dimensions (a result obtained independently by N. Tanaka), the first part gives a different solution, in terms of normal coordinates and corresponding normal forms.

Both approaches in [CM] start with analysis of homogeneous model hyperquadrics and their local symmetry groups. In the construction of normal forms the defining equation of the hypersurface is put into a form in which certain terms in its Taylor expansion vanish. In effect, the hypersurface is osculated by the model hyperquadric to a high order. Choosing the appropriate vanishing condition one can achieve that the transformation into normal form is determined uniquely, up to the action of the local symmetry group of the model hyperquadric. In the first step normal forms are obtained by algebraic manipulation of formal power series. The second step proves convergence of the transformation.

The results of Chern and Moser inspired a lot of subsequent work by many authors (e.g. $[\mathrm{F}],[\mathrm{J}],[\mathrm{V}],[\mathrm{B}],[\mathrm{W}],[\mathrm{EHZ}]$ and many others). Partial results in constructing normal forms on Levi degenerate hypersurfaces in $\mathbb{C}^{2}$ were obtained by Barletta-Bedford (hypersurfaces with a special symmetry), P. Wong (a subclass of hypersurfaces of type four) and N. Stanton (rigid hypersurfaces). P. Ebenfelt in $[\mathrm{E}]$ constructed normal forms for a class of hypersurfaces in $\mathbb{C}^{n}$ of finite type three.

The main difference, which seems inherent to degenerate hypersurfaces, is that normal forms are given by formal power series which need not converge (although we do not give any explicit example of a divergent normal form). The fact that a normal form construction solves the local equivalence problem relies on the essential result of Baouendi, Ebenfelt and Rothschild ([BER]), that any formal equivalence of two finite type hypersurfaces has to converge.

We give the first step in the construction of normal forms in section 2 . It is essentially a partial normalization which removes low order harmonic terms. This procedure reveals the type of the boundary point, an integer $k$, and a relatively simple substitute for the model hyperquadric, which plays a central role in Chern-Moser's theory. The fundamental information contained in the model hypersurface is its essential type, denoted by $l$. There are three qualitatively different types of model hypersurfaces, which have to be treated separately.

In section 3 we construct normal forms for the generic case, when $l<\frac{k}{2}$ and the model is not a tube. First we consider linear transformations and determine the local symmetry group of the model hypersurface, whose real dimension is equal to one. Normal form conditions are then defined (Definition 3.2) and shown to determine uniquely all coefficients of the biholomorphic transformation (Proposition 3.3). The remaining cases are considered in section 4. Definition 4.1 describes normal forms for the case of the most symmetric model hypersurface, when $l=\frac{k}{2}$. The symmetry group is three dimensional in this case. Definition 
4.3 gives normal forms for the case when the model hypersurface is a tube. Proposition 4.2 modifies Proposition 3.3 for these two cases.

Section 5 contains applications. First we show that the local equivalence problem is solved by the construction of normal forms combined with the convergence result of [BER] (Theorem 5.1). Then we apply our results to obtain precise information about the dimension of the stability group (Corollary 5.4).

I would like to thank Peter Ebenfelt, M. Salah Baouendi and Linda Preiss Rothschild for helpful comments on an earlier version of this paper.

\section{Hypersurfaces of finite type}

Let $M \subseteq \mathbb{C}^{2}$ be a real analytic hypersurface, and $p$ be a point on $M$. Let $r \in C^{\omega}$ be a local defining function, i.e., for a neighbourhood $U$ of $p$

$$
M \cap U=\{z \in U \mid r(z)=0\},
$$

and $\nabla r \neq 0$ in $M \cap U$. We recall a definition of a point of finite type. For a smooth real valued function $f$ defined in neighbourhood of 0 in $\mathbb{C}$ let $\nu(f)$ denote the order of vanishing of $f$ at 0 .

Definition 2.1. $p$ is a point of finite type, if there exists an integer $m$ such that

$$
\nu(r \circ \gamma) \leq m
$$

for all holomorphic maps $\gamma$ from a neighbourhood of $0 \in \mathbb{C}$ into $\mathbb{C}^{2}$, satisfying $\gamma(0)=p$ and $\gamma^{\prime}(0) \neq 0$. The smallest such integer is called the type of $p$.

Note that $M$ is Levi nondegenerate at $p$ if and only if $p$ is a point of finite type two. This is the case considered by Cartan and Chern-Moser. Hence, without any loss of generality, we will assume in the rest of this paper that $p$ is a point of finite type $k$, where $k>2$. Our aim is to assign to the pair $(M, p)$ formal power series in normal form. It will be unique up to the action of the symmetry group of the model hypersurface, which will be determined below.

Let $(z, w), z=x+i y, w=u+i v$, be local holomorphic coordinates centered at $p$ such that the hyperplane $\{v=0\}$ is tangent to $M$ at $p$. Near $p$, by the implicit function theorem, $M$ is described as a graph of a function

$$
v=F(x, y, u),
$$

where $F$ is a real valued function defined in a neighbourhood of the origin in $\mathbb{R}^{3}$. Since $r$ is real analytic, $F$ is the sum of its Taylor expansion starting with 2-nd order terms, which we express in terms of $(z, \bar{z}, u)$ :

$$
F(z, \bar{z}, u)=\sum_{i+j+m \geq 2} a_{i j m} z^{i} \bar{z}^{j} u^{m},
$$

where $a_{i j m}=\overline{a_{j i m}}$.

We will consider holomorphic transformations

$$
z^{*}=z+f(z, w), \quad w^{*}=w+g(z, w),
$$


where $f$ and $g$ are represented by power series

$$
f(z, w)=\sum_{i, j=0}^{\infty} f_{i j} z^{i} w^{j}, \quad g(z, w)=\sum_{i, j=0}^{\infty} g_{i j} z^{i} w^{j} .
$$

Since we will have to consider also formal hypersurfaces and formal transformations, from now on we allow both $F$ and $f, g$ to be formal power series. In this case (2.2) - (2.5) are interpreted in this sense.

We are interested only in transformations which preserve the above form, given by (2.2), (2.3). This will hold if and only if $f$ and $g$ contain no constant term, and $\frac{\partial v^{*}}{\partial x}, \frac{\partial v^{*}}{\partial y}, \frac{\partial v^{*}}{\partial u}$ are all zero at the origin. In other words, we require that

$$
f=0, \quad g=0, \quad g_{z}=0, \quad \operatorname{Im} g_{w}=0 \quad \text { at } \quad z=w=0 .
$$

In the following, we will consider only transformations satisfying (2.6). Let $F^{*}$ be the power series describing $M$ in new coordinates. Substituting (2.4) into $v^{*}=F^{*}\left(z^{*}, \bar{z}^{*}, u^{*}\right)$, we get the change of variables formula

$$
F^{*}(z+f, \bar{z}+\bar{f}, u+\operatorname{Re} g)=F(z, \bar{z}, u)+\operatorname{Im} g(z, u+i F(z, \bar{z}, u)),
$$

where $f$ and $R e g$ are also evaluated at $(z, u+i F(z, \bar{z}, u))$. It can be viewed as an equality of two power series in $z, \bar{z}, u$ which allows to obtain relations between the coefficients of $F^{*}$ and $F, f, g$.

The first step in our construction is the following standard result.

Lemma 2.2. There exist uniquely determined complex numbers $\alpha_{2}, \ldots, \alpha_{k}$ such that after the change of variable

$$
w^{*}=w+\sum_{i=2}^{k} \alpha_{i} z^{i}
$$

the defining equation (2.2) has form

$$
v^{*}=P(z, \bar{z})+o\left(|z|^{k}, u^{*}\right),
$$

where $P$ is a nonzero real valued homogeneous polynomial of degree $k$

$$
P(z, \bar{z})=\sum_{j=1}^{k-1} a_{j} z^{j} \bar{z}^{k-j},
$$

where $a_{j} \in \mathbb{C}$ and $a_{j}=\overline{a_{k-j}}$.

Proof. It follows from (2.7) that the value of $\alpha_{j}$ does not affect terms of order less than $j$ in $F^{*}$. We start with second order terms and write $F$ as

$$
F(z, \bar{z}, u)=A_{2} z^{2}+\bar{A}_{2} \bar{z}^{2}+a|z|^{2}+o\left(|z|^{2}, u\right) .
$$

After a change of variable $w^{*}=w+\sum_{i=2}^{k} \alpha_{i} z^{i}$, we obtain

$$
F^{*}\left(z, \bar{z}, u^{*}\right)=A_{2} z^{2}+\bar{A}_{2} \bar{z}^{2}+a|z|^{2}+\operatorname{Im} \alpha_{2} z^{2}+o\left(|z|^{2}, u^{*}\right) .
$$


Here $a$ is the value of the Levi form at $p$, so $a=0$. There is a unique $\alpha_{2}$ which makes the second order terms vanish, namely $\alpha_{2}=2 i A_{2}$. Now we proceed by induction. Let for some $j>2$ the coefficients $\alpha_{2}, \ldots, \alpha_{j-1}$ be already determined, so that

$$
F^{*}\left(z, \bar{z}, u^{*}\right)=P_{j}(z, \bar{z})+o\left(|z|^{j}, u^{*}\right)
$$

where $P_{j}(z, \bar{z})$ is a real valued homogeneous polynomial of degree $j$. Using Definition 2.1 it is easily verified that if $j<k$, we must have $P_{j}(z, \bar{z})=R e A_{j} z^{j}$ for some $A_{j} \in \mathbb{C}$. Hence we must take $\alpha_{j}=2 i A_{j}$. For $j=k, \alpha_{k}$ is uniquely determined by the requirement that $P$ in $(2.10)$ contains no harmonic term.

In order to preserve the form achieved by Lemma 2.1, all transformations which we will consider have to satisfy

$$
\frac{\partial^{j} g}{\partial z^{j}}=0 \text { at } z=w=0
$$

for $j=2, \ldots k$, in addition to $(2.6)$.

Let $l$ denote the lowest index in (2.10) for which $a_{l} \neq 0$. We have $1 \leq l \leq \frac{k}{2}$. Note that $l$ is the essential type of the model hypersurface to $M$ at $p$, defined below.

The problem now splits into three cases, depending on the form of $P$. Two cases are "exceptional", the case with extra symmetries, when $2 l=k$ and $P=$ $a_{l}|z|^{k}$, and the case when $P$ is equivalent to $(R e z)^{k}$, which corresponds to a tube domain. All other hypersurfaces will be treated together, as the generic case. We consider it first.

\section{Normal forms for generic models}

As a next step we consider the effect of a linear transformation

$$
w^{*}=\delta w, \quad z^{*}=\beta^{-1} z,
$$

where $\delta \in \mathbb{R}$ and $\beta \in \mathbb{C}$. Part of (3.1) will be used to normalize $P$, the other part will give the symmetry group of the model hypersurface.

In $(2.10)$ we have for $j<\frac{k}{2}$

$$
a_{j} z^{j} \bar{z}^{k-j}+a_{k-j} z^{k-j} \bar{z}^{j}=2|z|^{2 j} \operatorname{Re} a_{j} z^{k-2 j} .
$$

We will denote $j^{\prime}=k-2 j$ for $1 \leq j \leq \frac{k}{2}$, considering ' as an operator which can be applied to any integer from 1 to $\frac{k}{2}$. In order to normalize $P$ in the simplest possible way, we introduce the following notation. Let $l=m_{0}<m_{1}<\cdots<$ $m_{p}<\frac{k}{2}$ be the indices in $(2.10)$ for which $a_{m_{i}} \neq 0$. Denote by $L$ the greatest common divisor of $m_{0}^{\prime}, \ldots, m_{p}^{\prime}$ and let

$$
q_{i}=\frac{\operatorname{gcd}\left(m_{0}^{\prime}, \ldots, m_{i}^{\prime}\right)}{\operatorname{gcd}\left(m_{0}^{\prime}, \ldots, m_{i+1}^{\prime}\right)}
$$

for $0 \leq i \leq p-1$.

Lemma 3.1. There exists $\beta \in \mathbb{C}$ such that after the change of variables

$$
w^{*}=w, \quad z^{*}=\beta^{-1} z,
$$


and dropping stars, $P$ satisfies

$$
a_{l}=1
$$

and

$$
\arg a_{m_{i+1}} \in\left[0, \frac{2 \pi}{q_{i}}\right)
$$

for $0 \leq i \leq p-1$. These conditions determine $P$ uniquely, while $\beta$ is unique up to multiplication by an $L$-th root of unity.

Proof. The effect of (3.3) on (3.2) is simply multiplication of the coefficient by $|\beta|^{2 j} \beta^{k-2 j}$. The condition $a_{l}=1$ determines $\beta$ uniquely up to multiplication by an $l^{\prime}$-th root of unity. Each of the conditions (3.5) further reduces the number of possible values of $\beta$ by a factor of $q_{i}$. Since we have

$$
L \prod_{i=0}^{p-1} q_{i}=l^{\prime},
$$

there are $L$ possible values of $\beta$. On the other hand, if $\beta$ is an $L$-th root of unity, then (3.3) preserves $P$, and the conclusion follows.

We will denote by $M_{D}$ the model hypersurface at $p$ :

$$
M_{D}=\left\{(z, w) \in \mathbb{C}^{2} \mid v=P(z, \bar{z})\right\},
$$

which will be briefly called the model.

Up to now we have transformed $M$ into form (2.9), (2.10), (3.4), (3.5). The following transformations preserve the model:

$$
z^{*}=\delta e^{i \theta} z, \quad w^{*}=\delta^{k} w,
$$

where $e^{i \theta}$ is an $L$-th root of unity and $\delta>0$ for $k$ even or $\delta \in \mathbb{R} \backslash\{0\}$ for $k$ odd. Let $H$ denote the group of such transformations. Hence $H=\mathbb{R}^{+} \oplus \mathbb{Z}_{L}$ for $k$ even and $H=\mathbb{R}^{*} \oplus \mathbb{Z}_{L}$ for $k$ odd.

In the following we will assign weight 1 to $z, \bar{z}$ and weight $k$ to the variable $u$ in $F$ and $w$ in $f$ and $g$. Hence a monomial $z^{i} \bar{z}^{j} u^{m}$ has weight $i+j+k m$, and $z^{i} w^{j}$ has weight $i+k j$. Using weights we can write (2.9) as

$$
F=P+\text { terms of weight } \geq k+1,
$$

where $P$ satisfies (3.4) and (3.5).

We denote by $\mathcal{F}$ the set of formal power series of the form

$$
F(z, \bar{z}, u)=P(z, \bar{z})+\tilde{F}(z, \bar{z}, u),
$$

where

$$
\tilde{F}(z, \bar{z}, u)=\sum_{w t .>k} a_{i j m} z^{i} \bar{z}^{j} u^{m}
$$

We decompose $\tilde{F}$ into parts containing terms of equal weight:

$$
\tilde{F}=\sum_{\nu=k+1}^{\infty} F_{\nu} .
$$


We will also use partial expansion of $\tilde{F}$ in $z, \bar{z}$. Let

$$
Z_{i j}(u)=\sum_{m} a_{i j m} u^{m}
$$

so we can write

$$
\tilde{F}(z, \bar{z}, u)=\sum_{i, j} Z_{i j}(u) z^{i} \bar{z}^{j}
$$

Now we consider the group $\mathcal{T}_{0}$ of formal transformations preserving this form. It is easily verified that $\mathcal{T}_{0}$ consists of transformations of the form

$$
z^{*}=\delta e^{i \theta} z+\text { terms of weight } \geq 2, \quad w^{*}=\delta^{k} w+\text { terms of weight } \geq k+1,
$$

where again $e^{i \theta}$ is an $L$-th root of unity and $\delta>0$ for $k$ even or $\delta \in \mathbb{R} \backslash\{0\}$ for $k$ odd.

Let $\mathcal{T}$ be the set of formal transformations of the form

$$
\begin{aligned}
z^{*} & =z+\sum_{w t .>1} f_{i j} z^{i} w^{j} \\
w^{*} & =w+\sum_{w t .>k} g_{i j} z^{i} w^{j} .
\end{aligned}
$$

Clearly, $\mathcal{T}$ is a group under composition. Again we decompose the formal power series into parts of the same weight

$$
f=\sum_{\nu=2}^{\infty} f_{\nu} \quad \text { and } \quad g=\sum_{\nu=k+1}^{\infty} g_{\nu}
$$

and denote such an element of $\mathcal{T}$ by $(f, g)$.

If $F \in \mathcal{F}$ and $(f, g) \in \mathcal{T}$ it is easily verified using (2.7) that the formal power series resulting from transforming $F$ by $(f, g)$ is again in $\mathcal{F}$. Hence $\mathcal{T}$ acts on $\mathcal{F}$ via formula (2.7).

Now we check that any $\tau \in \mathcal{T}_{0}$ can be factored in a unique way as

$$
\tau=\phi \circ T
$$

with $\phi \in H$ and $T \in \mathcal{T}$. Here $\phi$ is simply the linear part of $\tau$. Hence we can use elements of $H$ to normalize transformations in $\mathcal{T}_{0}$ to satisfy

$$
f_{z}=0, \quad \operatorname{Re} g_{w}=0 \quad \text { at } z=w=0,
$$

i.e., to be in $\mathcal{T}$.

In summary, $\mathcal{T}$ consists precisely of transformations satisfying normalization conditions (2.6), (2.11) and (3.8).

For terms of weight $\mu>k$ in (2.7) we get from (2.7) and (3.6)

$$
\begin{array}{r}
F_{\mu}^{*}(z, \bar{z}, u)+2 \operatorname{Re} P_{z}(z, \bar{z}) f_{\mu-k+1}(z, u+i P(z, \bar{z}))= \\
=F_{\mu}(z, \bar{z}, u)+\operatorname{Im} g_{\mu}(z, u+i P(z, \bar{z}))+\ldots
\end{array}
$$

where dots denote terms depending on $f_{\nu-k+1}, g_{\nu}, F_{\nu}, F_{\nu}^{*}$ for $\nu<\mu$, and $P_{z}=$ $\frac{\partial P}{\partial z}$. 
The action of $\mathcal{T}$ defines an equivalence relation on $\mathcal{F}$ and our aim is to find a condition which selects a unique element in each class of equivalence. We will use the following scalar product on the vector space of homogeneous polynomials of degree $k-1$ without a harmonic term. If $Q=\sum_{j=1}^{k-2} \alpha_{j} z^{j} \bar{z}^{k-1-j}$ and $S=$ $\sum_{j=1}^{k-2} \beta_{j} z^{j} \bar{z}^{k-1-j}$, then

$$
(Q, S)=\sum_{j=1}^{k-2} \alpha_{j} \bar{\beta}_{j} .
$$

This notation will be used also for polynomials which may contain a harmonic term, which is then ignored. We need this notation also for polynomials whose coefficients depend on $u$. In particular, we denote

$$
\left(Z_{k-1}, P_{z}\right)=\sum_{j=1}^{k-2} Z_{j, k-1-j}(j+1) \bar{a}_{j+1} .
$$

Definition 3.2. We say that $F$ is in normal form if

$$
\begin{aligned}
Z_{j 0} & =0, \quad j=1,2, \ldots, \\
Z_{k-l+j, l} & =0, \quad j=0,1, \ldots, \\
Z_{2 k-2 l, 2 l} & =0, \\
\left(Z_{k-1}, P_{z}\right) & =0 .
\end{aligned}
$$

We will prove

Proposition 3.3. For any $F \in \mathcal{F}$ there is a uniquely determined formal transformation $T \in \mathcal{T}$ which transforms $F$ into normal form.

Proof. By induction on weight we show that the condition that $F^{*}$ satisfy (3.11) determines uniquely all coefficients of $f$ and $g$ in (3.7). Let us consider terms of weight $\mu$ in (2.7). For an analytic function $\phi$ of two variables we use the identity

$$
\phi(z, u+i P(z, \bar{z}))=\sum_{n=0}^{\infty} i^{n} \frac{\phi^{(n)}(z, u)}{n !} P(z, \bar{z})^{n},
$$

where $\phi^{(n)}$ denotes the $n$-th derivative of $f$ with respect to $u$. We shall need this expansion up to the third order. Denoting derivatives with respect to $u$ by primes, we have

$$
\phi(z, u+i P)=\phi(z, u)+i \phi^{\prime}(z, u) P-\frac{1}{2} \phi^{\prime \prime}(z, u) P^{2}-\frac{i}{6} \phi^{\prime \prime \prime}(z, u) P^{3}+\ldots
$$

In (3.9) we denote

$$
L(f, g)=\operatorname{Re}\left\{i g(z, u+i P(z, \bar{z}))+2 P_{z} f(z, u+i P(z, \bar{z}))\right\} .
$$


From (3.12) we have

$$
\begin{aligned}
2 L(f, g) & =2 f P_{z}+2 i f^{\prime} P_{z} P-f^{\prime \prime} P_{z} P^{2}+2 \bar{f} \bar{P}_{z}-2 i \bar{f}^{\prime} \bar{P}_{z} \bar{P}-\bar{f}^{\prime \prime} \bar{P}_{z} \bar{P}^{2} \\
& +i g-g^{\prime} P-\frac{i}{2} g^{\prime \prime} P^{2}+\frac{1}{6} g^{\prime \prime \prime} P^{3}-i \bar{g}-\bar{g}^{\prime} \bar{P}+\frac{i}{2} \bar{g}^{\prime \prime} \bar{P}^{2}+\frac{1}{6} \bar{g}^{\prime \prime \prime} \bar{P}^{3}+\ldots
\end{aligned}
$$

In this expansion we will collect terms of type $(i, j)$.

In order to compute the coefficients of terms of the types specified by (3.11), let us denote $F_{i j}^{*}=a_{i j m}^{*}$ and $F_{i j}=a_{i j m}$ if $i+j+k m=\mu, f_{i}=f_{i j}$ if $i+k j=$ $\mu-k+1$ and $g_{i}=g_{i j}$ if $i+k j=\mu$. Similarly, for the derivatives of $f(z, u)$ and $g(z, u)$ with respect to $u$, we denote $f_{i}^{\prime}=j f_{i j}, f_{i}^{\prime \prime}=j(j-1) f_{i j}$ if $i+k j=\mu-k+1$ and $g_{i}^{\prime}=j g_{i j}, g_{i}^{\prime \prime}=j(j-1) g_{i j}, g_{i}^{\prime \prime \prime}=j(j-1)(j-2) g_{i j}$, if $i+k j=\mu$. To separate this notation from the one used for weights, subscripts indicating weight are always greek letters. Finally, in analogy to (3.10) we write

$$
\left(F_{k-1}, P_{z}\right)=\sum_{j=1}^{k-2} F_{j, k-1-j}(j+1) \bar{a}_{j+1},
$$

and the same for $F^{*}$. For $j \geq 1$ and $j \neq k-1$ we have from (3.9) and (3.13)

$$
F_{j 0}^{*}=-\frac{i}{2} g_{j}+F_{j 0}+\ldots
$$

Here dots denote terms with values already determined, i.e. terms depending on $f_{\nu-k+1}, g_{\nu}, F_{\nu}, F_{\nu}^{*}$ for $\nu<\mu$. For $j=k-1$ we have

$$
F_{k-1,0}^{*}=-\frac{i}{2} g_{k-1}-\bar{f}_{0} \delta_{1 l}+F_{k-1,0}+\ldots,
$$

where $\delta_{1 l}$ is Kronecker's delta. Further, for $j \geq 1$ and $j \neq k-1$ only the first and the eighth term in (3.13) contribute to type $(k-l+j, l)$, and we get

$$
F_{k-l+j, l}^{*}=-(k-l) f_{j+1}+\frac{1}{2} g_{j}^{\prime}+F_{k-l+j, l}+\ldots
$$

For $j=k-1$ we have

$$
F_{2 k-l-1, l}^{*}=-(k-l) f_{k}+\frac{1}{2} g_{k-1}^{\prime}+i \bar{f}_{0} \delta_{1 l}+\ldots
$$

Next

$$
F_{k-l, l}^{*}=-(k-l) f_{1}-l \bar{f}_{1}+R e g_{0}^{\prime}+F_{k-l, l}+\ldots
$$

and

$$
\begin{aligned}
& F_{2 k-2 l, 2 l}^{*}=-(k-2 l) f_{k+1} \bar{a}_{2 l}-i(k-l) f_{1}^{\prime}+i l \bar{f}_{1}^{\prime}+ \\
& \frac{1}{2} g_{k}^{\prime} \bar{a}_{2 l}-\frac{1}{2} I m g_{0}^{\prime \prime}+F_{2 k-2 l, 2 l}+\ldots
\end{aligned}
$$

Further,

$$
\left(F_{k-1}^{*}, P_{z}\right)=f_{0}\left(P_{z}, P_{z}\right)+\bar{f}_{0}\left(P_{\bar{z}}, P_{z}\right)+\left(F_{k-1}, P_{z}\right)+\ldots
$$


We first show that (3.21) determines uniquely $f_{0}$. For this we use the elementary fact that an equation $\alpha f_{0}+\beta \bar{f}_{0}=\gamma$, where $\alpha, \beta, \gamma \in \mathbb{C}$, determines uniquely $f_{0}$ if and only if $|\alpha| \neq|\beta|$. Since $\bar{P}_{\bar{z}}=P_{z}$, we have $\left(P_{z}, P_{z}\right)=\left(P_{\bar{z}}, P_{\bar{z}}\right)$. By Cauchy-Schwartz inequality

$$
\left|\left(P_{z}, P_{\bar{z}}\right)\right| \leq\left\{\left(P_{\bar{z}}, P_{\bar{z}}\right)\left(P_{z}, P_{z}\right)\right\}^{\frac{1}{2}}=\left(P_{z}, P_{z}\right),
$$

with equality if and only if $P_{z}$ is a scalar multiple of $P_{\bar{z}}$, i.e., $P_{z}=e^{i \theta} P_{\bar{z}}$, modulo harmonic terms. It is easy to verify that this happens if and only if $P$ is equivalent to $(R e z)^{k}$, the exceptional case treated in section 4. Next, (3.15) and (3.16) determine $g_{j}$ for all $j \geq 1$. Then (3.17) and (3.18) determine $f_{j}$ for all $j \geq 2$. The pair of equations (3.19), (3.20) then determines $f_{1}$ and $g_{0}$. It is straightforward to verify that the initial appearence of each equation agrees with our normalization conditions for $f$ and $g$.

\section{Normal forms for exceptional models}

Now we consider the remaining two cases. In the first one, when $2 l=k$, the model hypersurface is described by

$$
v=|z|^{k} .
$$

It is preserved by transformations of the form

$$
z^{*}=\frac{\delta e^{i \theta} z}{(1+\mu w)^{\frac{1}{l}}}, \quad w^{*}=\frac{\delta^{k} w}{1+\mu w},
$$

where $\delta>0$, and $\theta, \mu \in \mathbb{R}$. We denote this group again by $H$. Its real dimension is equal to three. We consider hypersurfaces of the form

$$
v=|z|^{k}+\text { terms of weight } \geq k+1 .
$$

Now we define $\mathcal{F}$ as in section 3 . Again, $\mathcal{T}_{0}$ will denote the group of transformations which preserve this form. Its elements are of the form

$$
z^{*}=\delta e^{i \theta} z+\text { terms of weight } \geq 2, \quad w^{*}=\delta^{k} w+\text { terms of weight } \geq k+1,
$$

where $\theta \in \mathbb{R}$ and $\delta \in \mathbb{R}^{+}$.

$\mathcal{T}$ will denote the group of transformations of the form (3.7), satisfying an additional normalization condition

$$
\text { Re } g_{w w}=0 .
$$

It is verified easily that we can factor any $\tau \in \mathcal{T}_{0}$ uniquely as $\tau=\phi \circ T$, where $\phi \in H$ and $T \in \mathcal{T}$. Indeed, the additional condition determines $\mu$ in (4.1), while $\delta$ and $e^{i \theta}$ are determined by (3.8). 
Definition 4.1. We say that $F$ is in normal form if

$$
\begin{aligned}
Z_{j 0} & =0, \quad j=0,1, \ldots, \\
Z_{l, l+j} & =0, \quad j=1,2, \ldots, \\
Z_{l, l} & =0, \\
Z_{2 l, 2 l} & =0, \\
Z_{3 l, 3 l} & =0, \\
Z_{2 l, 2 l-1} & =0 .
\end{aligned}
$$

We have

Proposition 4.2. For any $F \in \mathcal{F}$ there is a uniquely determined formal transformation $T \in \mathcal{T}$ which transforms $F$ into normal form.

Proof. With the notation introduced in the proof of Proposition 3.3, we obtain for $j \geq 0$ from (3.9) and (3.13)

$$
\begin{aligned}
& F_{j 0}^{*}=-\frac{i}{2} g_{j}+F_{j 0}+\ldots \\
& F_{00}^{*}=\operatorname{Im} g_{0}+F_{00}+\ldots
\end{aligned}
$$

For $j \geq 1$ we have

$$
\begin{aligned}
F_{l l}^{*} & =\operatorname{Re} g_{0}^{\prime}-2 l \operatorname{Re} f_{1}+F_{l l}+\ldots \\
F_{l+j, l}^{*} & =-l f_{j+1}+\frac{1}{2} g_{j}^{\prime}+F_{l+j, l}+\ldots \\
F_{2 l, 2 l}^{*} & =-\frac{1}{2} \operatorname{Im} g_{0}^{\prime \prime}+2 l \operatorname{Im} f_{1}^{\prime}+F_{2 l, 2 l}+\ldots \\
F_{3 l, 3 l}^{*} & =-\frac{1}{6} \operatorname{Re} g_{0}^{\prime \prime \prime}+l \operatorname{Re} f_{1}^{\prime \prime}+F_{3 l, 3 l}+\ldots \\
F_{2 l, 2 l-1}^{*} & =-i l \bar{f}_{0}^{\prime}+F_{2 l, 2 l-1}+\ldots
\end{aligned}
$$

Again, the condition that $F^{*}$ be in normal form determines uniquely coefficients of $f$ and $g$. Equations (4.3) determine $g_{j}$ for all $j \geq 1$ and $I m g_{0}$. The second equation in (4.4) determines $f_{j}$ for $j \geq 2$ and the fifth $f_{0}$. The third equation determines $\operatorname{Im} f_{1}$. Finally the first and the fourth equations determine $R e f_{1}$ and $\operatorname{Re} g_{0}$.

Now we consider the model hypersurface equivalent to $\left\{v=(\operatorname{Re} z)^{k}\right\}$. In this case

$$
P(z, \bar{z})=\frac{1}{k}\left[(z+\bar{z})^{k}-2 \operatorname{Re} z^{k}\right] .
$$

Definition 4.3. We say that $F$ is in normal form if

$$
\begin{aligned}
Z_{j 0} & =0, \quad j=1,2, \ldots, \\
Z_{k-l+j, l} & =0, \quad j=0,1, \ldots, \\
Z_{2 k-2 l, 2 l} & =0, \\
\operatorname{Re} Z_{k-2,1} & =0, \\
\operatorname{Re} Z_{k, k-1} & =0 .
\end{aligned}
$$


$\mathcal{T}_{0}$ and $\mathcal{T}$ are now defined as for generic models in part 3. Now we prove Proposition 4.2 for this case.

Proof of Proposition 4.2. We proceed as in the proof of Proposition 3.3. Instead of (3.21) we obtain

$$
\text { Re } F_{k-2,1}^{*}=-2(k-1) \operatorname{Re} f_{0}+\operatorname{Re} F_{k-2,1}+\ldots
$$

and

$$
\operatorname{Re} F_{k, k-1}^{*}=(2 C-1) \operatorname{Im} f_{0}+\frac{1}{2} \operatorname{Re} g_{k-1}^{\prime}+\operatorname{Re} F_{k, k-1},
$$

where $C=\frac{1}{k}\left[\left(\begin{array}{c}2 k-1 \\ k\end{array}\right)-1\right]$ is the coefficient of $z^{k} \bar{z}^{k-1}$ in $P P_{\bar{z}}$. Since $k \geq 3$, we have $C \geq 3$, and this pair of equations together with (3.16) determine uniquely $g_{k-1}$ and $f_{0}$.

\section{The equivalence problem and applications}

Propositions 3.3 and 4.2 combined with the result of [BER] give a solution to the local equivalence problem. Let $\mathcal{N}$ be the subset of $\mathcal{F}$ containing formal power series in normal form. Let $M$ be a hypersurface described by (3.6) (where the exceptional cases of $\mathrm{P}$ are now included), and let $T_{0} \in \mathcal{T}_{0}$ be a transformation which takes $M$ into normal form. Writing $T_{0}=\phi \circ T$, with $\phi \in H$ and $T \in \mathcal{T}$, we see from Propositions 3.3 and 4.2 that transformations into normal form are parametrized by elements of $H$. If $M$ is already in normal form, we get a natural action of $H$ on $\mathcal{N}$. It is obtained by first applying an element of $H$ and then renormalizing by a unique element in $\mathcal{T}$.

Since normal forms are unique up to this action of $H$, we consider the set of equivalence classes $\mathcal{N} \bmod H$ and call the elements of this set normal forms.

Theorem 5.1. Two real analytic hypersurfaces are locally biholomorphically equivalent if and only if their normal forms are equal.

Proof. Clearly the normal form is a biholomorphic invariant. On the other hand, if $\phi_{1}, \phi_{2}$ are formal transformations of $M_{1}, M_{2}$ respectively into the same power series in normal form, then $\phi_{1} \circ \phi_{2}^{-1}$ is a formal equivalence of $M_{1}, M_{2}$. Since the hypersurfaces are of finite type, the result of $[\mathrm{BER}]$ implies that it converges.

For nondegenerate hypersurfaces it may be quite difficult to decide whether two hypersurfaces in normal form are equivalent under the action of $H$, which has real dimension five. In the degenerate case, this becomes much simpler. As an example, let us consider the following case. Let $M_{1}, M_{2}$ be two hypersurfaces of finite type $k$ in normal form, which for $M_{1}$ is given by

$$
P(z, \bar{z})+\sum_{i+j<k-1} \sum_{m} a_{i j m} z^{i} \bar{z}^{j} u^{m}
$$

and for $M_{2}$ by

$$
P(z, \bar{z})+\sum_{i+j<k-1} \sum_{m} b_{i j m} z^{i} \bar{z}^{j} u^{m}
$$


where $P$ is a polynomial of the form $(2.10),(3.4),(3.5)$ and the sums on the right contain only terms of weight bigger than $k$. We have the following consequence of Proposition 3.3.

Proposition 5.2. $M_{1}$ and $M_{2}$ are locally biholomorphically equivalent if and only if there is $\left(\delta, e^{\sqrt{-1} \theta}\right) \in H$ such that

$$
a_{i j m}=\delta^{i+j+k m} e^{\sqrt{-1}(i-j) \theta} b_{i j m}
$$

for all indices $(i, j, m)$.

As a corollary of Propositions 3.3 and 4.2 we obtain

Corollary 5.3. The only transformations which preserve the model hypersurface are the elements of $H$.

Proof. Let $\tau \in \mathcal{T}_{0}$ be a transformation which preserves the model hypersurface. There is a unique $\phi \in H$ such that $\phi \circ \tau$ is an element of $\mathcal{T}$. This mapping still preserves the model hypersurface, in particular it is a mapping into normal form. By Propositions 3.3 and 4.2, $\phi \circ \tau$ is the identity, therefore $\tau$ is an element of $H$.

Propositions 3.3 and 4.2 also give immediately precise information about the dimension of the stability group (the local symmetry group) of $M$ at $p$.

Corollary 5.4. Let $M$ be a real analytic hypersurface in $\mathbb{C}^{2}$ and let $p \in M$ be a point of finite type $k$, where $k \geq 3$. Then the dimension of the stability group of $M$ at $p$ is less or equal to three. If, moreover, the model hypersurface at $p$ is different from $\left\{v=|z|^{k}\right\}$, then the dimension is at most one.

\section{References}

[BER] M. S. Baouendi, P. Ebenfelt, and L. P. Rothschild, Convergence and finite determination of formal CR mappings, J. Amer. Math. Soc. 13 (2000) 697-723.

[BB] E. Barletta and E.Bedford, Existence of proper mappings from domains in $\mathbb{C}^{2}$, Indiana Univ. Math. J. 39 (1990) 315-338.

[BFG] M. Beals, C. Fefferman, and R. Grossman, Strictly pseudoconvex domains in $\mathbb{C}^{n}$, Bull. Amer. Math. Soc. (N.S.) 8 (1983) 125-322.

[B] V. K. Beloshapka, On the dimension of the group of automorphisms of an analytic hypersurface, Izv. Akad. Nauk SSSR Ser. Mat. 43 (1979) 243-266.

[C1] E. Cartan, Sur la géométrie pseudo-conforme des hypersurfaces de deux variables complexes, I, Ann. Math. Pura Appl. 11 (1932) 17-90.

[C2] Sur la géométrie pseudo-conforme des hypersurfaces de deux variables complexes, II, Ann. Scoula Norm. Sup. Pisa 1 (1932) 333-354.

[CS] S.-C. Chen and M.-C. Shaw, Partial differential equations in several complex variables, American Mathematical Society/International Press, 2001.

[CM] S. S. Chern and J. Moser, Real hypersurfaces in complex manifolds, Acta Math. 133 (1974) 219-271.

[E] P. Ebenfelt, New invariant tensors in CR structures and a normal form for real hypersurfaces at a generic Levi degeneracy, J. Differential Geom. 50 (1998) 207-247.

[EHZ] P. Ebenfelt, X. Huang, and D. Zaitsev, The equivalence problem and rigidity for hypersurfaces embedded into hyperquadrics, Amer. J. Math. 127 (2005) 169-191.

[F] C. Fefferman, Parabolic invariant theory in complex analysis, Adv. Math. 31 (1979) $131-262$. 
[J] H. Jacobowitz, An introduction to CR structures, Mathematical Surveys and Monographs 32, Amer. Math. Soc., 1990.

[K] J. J. Kohn, Boundary behaviour of $\bar{\partial}$ on weakly pseudoconvex manifolds of dimension two, J. Differential Geom. 6 (1972) 523-542.

[S] N. Stanton, A normal form for rigid hypersurfaces in $\mathbb{C}^{2}$, Amer. J. Math. 113 (1991) 877-910.

[V] A. G. Vitushkin, Real analytic hypersurfaces in complex manifolds, Russ. Math. Surv. 40 (1985) 1-35.

[W] S. M. Webster, On the Moser normal form at a non-umbilic point, Math. Ann. 233 (1978) 97-102.

[Wo] P. Wong, A construction of normal forms for weakly pseudoconvex $C R$ manifolds in $\mathbb{C}^{2}$, Invent. Math. 69 (1982), 311-329.

Department of Mathematical Analysis, Masaryk University, Janackovo nam. 2A, 66295 BRNO

E-mail address: mkolar@math.muni.cz 